\title{
Quantised Inertia and Galaxy Rotation from Information Theory
}

\author{
M. E. McCulloch \\ University of Plymouth, PL4 8AA, UK \\ Email: mike.mcculloch@plymouth.ac.uk
}

\begin{abstract}
The theory of quantised inertia (QI) can predict galaxy rotations without dark matter. It is shown here that QI can be derived from Landauer's principle by assuming that bits of information are stored in the distances between objects and the horizons they see.
\end{abstract}

Keywords: Quantised inertia, Information, Galaxy rotation.

\section{Introduction}

Many astrophysical observations show that stars at the outer edges of galaxies orbit far too fast to be gravitationally bound to the galaxy $([1],[2])$ and an identical phenomenon is observed for globular clusters ([3]) and wide binaries ([4]). A good summary of these and similar anomalies can be found in [5]. In galaxies and galaxy clusters this has been typically corrected ad hoc by adding dark matter, whose arbitrariness is unsatisfactory (It should be noted that dark matter is also widely used to model the big bang cosmology, baryon acoustic oscillations and other observations).

On a much smaller scale, some laboratory experiments have shown that asymmetric metal cavities of various types with strong electromagnetic fields resonating within them show an unexpected thrust towards their narrower ends ([6], [7]).

These large and small scale phenomena can be explained by a theory called quantised inertia [8]. When an object accelerates, say, to the right, a so-called Rindler horizon appears to its left as information, limited to the speed of light, cannot catch up to the object from beyond that horizon. In the same way that black holes are assumed to radiate Hawking radiation, these horizons radiate Unruh radiation. Quantised inertia says that the inertial force on an object arises because this Rindler horizon damps (reduces) the excited zero point field (Unruh radiation) on the side of the object opposite to its acceleration vector, creating an Unruh radiation imbalance which pushes it back against its original acceleration ([9]). The modified inertia $m^{\prime}$ is given by

$$
m^{\prime}=m\left(1-\frac{2 c^{2}}{a \Theta}\right)
$$

where $m$ is the unmodified mass, $c$ is the speed of light, $a$ is the acceleration and $\Theta=8.8 \times 10^{26} m$ is the cosmic scale. Eq. 1 successfully predicts galaxy rotations ([10]) and wide binary orbits ([11]) without any dark matter and without any adjustment. Quantised inertia also predicts that an artificial horizon can be made when high acceleration matter or light is confined inside an asymmetric cavity, producing a new kind of thrust ([12]). The theory is not yet mathematically complete as pointed out by [13], and also, attributing inertial mass to the push from virtual photons simply pushes the inertia problem further back. How then do the photons gain inertia?

An alternative approach may be possible using information theory. Landauer [14] pointed out that when computer memory is erased, for example the binary number 101011 is reset to be 000000 , then this represents a real, and hitherto ignored, reduction of the entropy of the internal device. Entropy cannot decrease, by the second law of thermodynamics, and so this erasure of information must be accompanied by a release of thermal energy to ensure a global entropy increase.

Landauer's principle was used to model dark energy by [15] who argued that the deletion of space as the cosmic horizon expands releases the required energy. An alternative and independent method of deriving quantised inertia from Landauer's principle was suggested by [16] in which Planck-areas of 
horizons represent bits and release energy upon the shrinking of the horizon to power the phenomenon of inertia, but the derivation was complex and it was only possible to derive Eq. 1 to within $8 \%$. This can be corrected by, as done by [17], making the horizon width an important factor instead of area, but there is no good justification for using the width instead of the area.

[18] and [19] argued that if mass is associated with bits in computer systems then changes in mass might be detactable when data is erased. These works re-emphasise Landauer's point that information should be physical, and not stored on abstract horizons.

This paper presents a successful derivation of quantised inertia from Landauer's principle, building on [16] but assuming that information is not stored in the abstract horizon but is stored in the space between the object and the horizon it sees.

\section{Method}

According to Landauer's principle [14] the mass-energy released $(d E)$ from the deletion of one bit of information can be given by

$$
d E=k T \log _{2} 2
$$

Here the usual natural logarithm has been replaced with a log to the base of $2\left(\log _{2}\right)$. The first reason for this is that the use of natural log was based on mathematical considerations and not physical ones. For example the derivative of $\ln (x)$ is simpler than that of $\log (x)$. The second reason is that the use of $\log _{2}$ is more consistent with information entropy (eg [20]). Also, experimental tests of the principle are ambiguous enough to allow both (see Fig. 4 of [21]). For $N$ bits we have an energy content of

$$
d E=k T N \log _{2} 2=k T N=m c^{2}
$$

Figure 1 shows an object extended along the y (vertical axis) (the dashed vertical line on the left) and accelerating to the left (see arrow). The cosmic horizon seen by the object before it accelerated is shown by the vertical line on the right. The closer horizon seen after its acceleration ' $a$ ' is shown by the vertical line in the centre. Here, we only have to consider changes to the horizon on the right since the horizon on the left, in the direction of acceleration, is unchanged by the acceleration. Now, there is a difference in energy available to the object because the horizon it sees has moved closer, so the number of bits stored in the space it can see has decreased from $N_{1}$ to $N_{2}$ and this has destroyed some information and entropy. To satisfy the second law of thermodynamics, it must release energy $d E$

$$
d E=k T N_{1}-k T N_{2}=m c^{2}
$$

The number of bits stored in the space, the number $N$, is calculated as the number of Planck lengths to the horizon (the minimum observable distance in nature so the minimum distance that can contain information). The number of bits to the cosmic horizon is

$$
N_{1}=\frac{\Theta}{2 l_{P}}
$$

and for the new, smaller Rindler horizon we get

$$
N_{2}=\frac{d_{R}}{l_{P}}=\frac{c^{2}}{a l_{P}}
$$

Substituting Eqs. 5 and 6 into Eq. 4, and using $m=E / c^{2}$ we get

$$
M=\frac{k T \Theta}{2 c^{2} l_{P}}\left(1-\frac{2 c^{2}}{a \Theta}\right)
$$

Assuming the energy is thermalised $E=k T$ and $E=m c^{2}$ so

$$
M=m \frac{\Theta}{2 l_{P}}\left(1-\frac{2 c^{2}}{a \Theta}\right)
$$




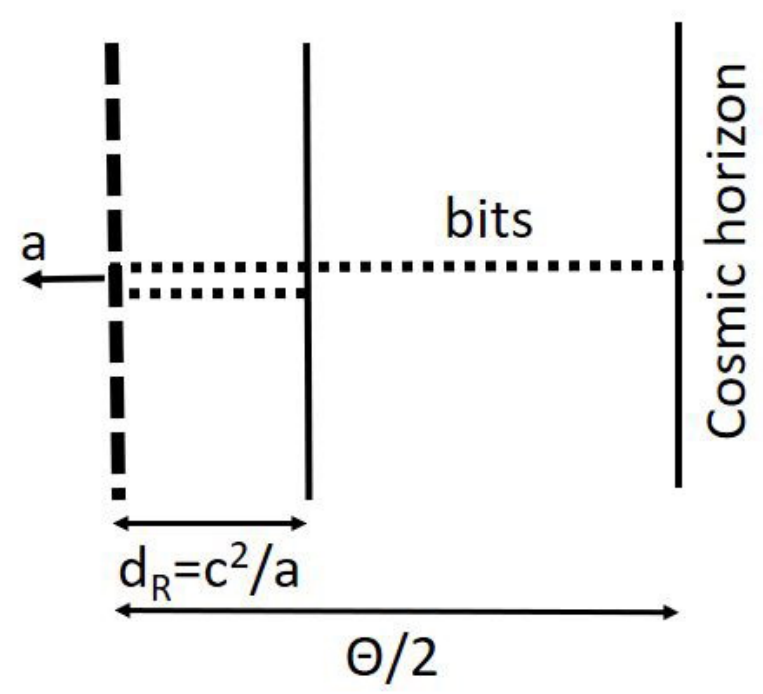

Figure 1. Schematic of an object (thick dashed line on the left) that initially sees the cosmic horizon and many bits of space. When it accelerates to the left (arrow) it sees a much closer Rindler horizon (middle vertical line) and some spatial bits are erased. To ensure entropy increases, energy is released to oppose the acceleration: exactly the amount required for quantised inertia.

$M$ is the predicted mass for the extended object, which has a length of $\Theta / 2 l_{P}$ Planck lengths. For a particle with a width of one Planck length, we divide by this quantity and get the mass $m^{\prime}$

$$
m^{\prime}=m\left(1-\frac{2 c^{2}}{a \Theta}\right)
$$

This is the formula for mass in quantised inertia, which explains galaxy rotation without dark matter as shown by [10], and predicts propellant-less propulsion [12].

\section{Discussion}

This model implies that the energy required to provide the phenomenon known as inertia, a push-back against acceleration, comes from the destruction of information as space is hidden by horizons upon higher accelerations. As shown here, the energy released is exactly that required to predict standard inertial mass plus the slight deviation from it that enables quantised inertia to predict galaxy rotation without dark matter. The derivation is simple and elegant.

There are now three ways to derive quantised inertia. Firstly by assuming that horizons make a gradient in Unruh radiation [9]. Second, by using the uncertainty principle [22] and third from information theory, as done here. All three methods predict Eq. 1 for the modified mass.

\section{Conclusion}

Quantised inertia correctly predicts galaxy rotations without dark matter or adjustment, and predicts an application: propellant-less thrust.

It is shown here that quantised inertia can be derived from information theory by assuming that bits of information are stored in space and are erased, releasing energy, when information horizons form upon acceleration.

Acknowledgements. Thank you to the reviewer for their useful comments and to DARPA for grant HR001118C0125. 


\section{References}

1. Zwicky, F., 1937. On the masses of nebulae and clusters of nebulae. Astrophysical Journal, 86, 217.

2. Rubin, V.C., W.K. Ford, 1970. The Astrophysical Journal, 159, 379.

3. Scarpa, R., G. Marconi, R. Gimuzzi and G. Carraro, 2007. A $\& A$, 462, L9.

4. Hernandez, X., R.A.M. Cortes, C. Allen and R. Scarpa, 2018. Challenging a Newtonian prediction through Gaia wide binaries. IJMP-D, 28, 8, 1950101

5. Unzicker, A., 2008. Why do we still believe in Newton's law? Facts, myths and methods in gravitational physics. arXiv: gr-qc/0702009.

6. Shawyer, R, 2008. Microwave propulsion - progress in the emdrive programme. 59th International Astronautical Conference. IAC-2008. Glasgow, UK.

7. White, H., P. March, J. Lawrence, J. Vera, A. Sylvester, D. Brady and P. Bailey, 2016. J. Propulsion and Power. doi:10.2514/1.B36120.

8. McCulloch, M.E., 2007. Modelling the Pioneer anomaly as modified inertia. Mon. Not. Roy. Astro. Soc., 376, 338-342.

9. McCulloch, M.E., 2013. Inertia from an asymmetric Casimir effect. EPL, 101, 59001.

10. McCulloch, M.E., 2012. Testing quantised inertia on galactic scales. Astro. Sp. Sci., 342, 2, 575-578.

11. McCulloch, M.E. \& J. Lucio, 2019. Testing Newton/GR, MoND \& quantised inertia on wide binaries. ApSS., 364,121

12. McCulloch, M.E., 2017. Testing quantised inertia on emdrives with dielectrics. EPL, 118, 34003.

13. Renda, M., 2019. A sceptical analysis of quantised inertia. MNRAS, 489, 1, 881-885.

14. Landauer, R., 1961. Irreversibility and heat generation in the computing process. IBM J. Research and Development. 5(3), 183-191.

15. Lee J-W, J.J Lee and H.C. Kim, 2007. Quantum informational dark energy: dark energy from forgetting. Proc. Nat. Inst. for Math. Science, 8, 1.

16. McCulloch, M.E. and J. Gine, 2017. Modified inertial mass from information loss. MPL-A, 32, 28, 1750148.

17. McCulloch, M.E. and J. Gine, 2020. Deriving quantised inertia using horizon-widths in the uncertainty principle. $A S T P, 14,1,1-8$.

18. Kish, L.B., 2007. "Gravitational mass" of information? Fluctuation and Noise Letters, 2007, Vol: 7 Issue: 4 , C51 - C68.

19. Vopson, M.M., 2019. The mass-energy-information equivalence principle. AIP Advances, 9, 095206.

20. Shannon, C.F., 1948. A mathematical theory of communication. The Bell System Technical Journal. 27, 379-423.

21. Hong, J., B. Lambson, S. Dhuey and J. Bokor, 2016. Experimental test of Landauer's principle in single-bit operations on nanomagnetic memory bits. Science Advances, Vol. 2, no. 3, e1501492.

22. McCulloch, M.E., 2016. Quantised inertia from relativity and the uncertainty principle. EPL, 115, 69001. 